\title{
Matriosche: nonne, madri e nipoti. Tre esempi di genealogie femminili nella letteratura italiana del Novecento
}

\author{
Matriosche: grandmothers, mothers and granddaughters. \\ Three examples of female genealogies \\ in Italian literature of twentieth
}

\author{
Daniele Cerrato \\ Università di Siviglia \\ dcerrato@us.es
}

\begin{abstract}
Starting from the myth, culture and society have always opposed the construction of a matriarchal line and a genealogy of women. The paper address the generational relationships between women, and focuses on three novel Italian Novecento women writers: Susanna Tamaro, Michela Murgia and Milena Agus. Tamaro, Murgia and Agus conform an atypical female genealogy in their novels, and propose new models of identity in opposition to the patriarchal system.
\end{abstract}

Keywords: Women writers, genealogy, female subjectivity, mothers, Italian literature

L'obiettivo del presente articolo è riflettere sul concetto di genealogia femminile ed indagare le relazioni tra nonne, madri e nipoti a partire dai testi di tre autrici italiane contemporanee: Susanna Tamaro, Michela Murgia e Milena Agus.

Il titolo matriosche fa riferimento a figure che ne contengono altre ma al tempo stesso possono considerarsi pezzi a sé stanti e, quindi, indipendenti in una genealogia che si compone e scompone e dove i rapporti generazionali possono venire stravolti e capovolti. Dalla 'madre', così come si chiama la matriosca più grande, si può passare direttamente al 'seme', la matriosca più piccola, che non ne contiene altre, ma è quella da cui tutto si genera, in una relazione di continua reciprocità. 
C'è una genealogia di donne nella nostra famiglia: abbiamo una madre, una nonna, una bisnonna materne e delle figlie. Di questa genealogia di donne, dato il nostro esilio nella famiglia del padre marito, tendiamo a dimenticarne la singolarità e perfino a rinnegarla. Cerchiamo di situarci in questa genealogia femminile per conquistare e custodire la nostra identità (Irigaray, 1989, 30).

Come sottolinea Luce Irigaray, la costruzione della soggettività femminile passa necessariamente dalla creazione di una personale genealogia, diversa rispetto a quella regolata attraverso un ordine patriarcale. L'assenza di modelli specifici di riferimento e di una genealogia femminile, hanno infatti reso difficile l'accesso delle donne ad una propria identità, circondandole solamente di archetipi costruiti e imposti dalla società e dalla cultura patriarcale.

La volontà di privare le donne di una linea matriarcale si avverte fin dai miti classici che fanno riferimento al rapporto madre-figlia e mostrano una genealogia femminile discontinua e frammentaria ${ }^{1}$. Come osserva Rodriguez Magda (1999), la struttura patriarcale si basa, infatti, proprio sull'idea di genealogia, poiché ha come finalità l'unità della famiglia, l'inclusione in un lignaggio, i vincoli di sangue e i patti economici e di difesa con altre famiglie e clan ${ }^{2}$. Uno degli obiettivi che la maggior parte dei miti greci si propone, è proprio quello di cancellare e rovesciare la mitologia precedente legata alla Grande Madre, in cui le divinità femminili ricoprono ruoli di primo piano. L'esempio più noto di linea matriarcale e di rapporto madre/figlia interrotto, ma poi ricostituito, è il mito di Demetra e Persefone (o Core) a cui fanno riferimento anche Irigaray e Muraro ${ }^{3}$. Persefone viene rapita e stuprata dallo zio Ade, che la vuole come sposa, tutto questo con il beneplacito del padre di

\footnotetext{
${ }^{1}$ In varie tragedie il vincolo madre-figlia si spezza attraverso il rituale del sacrificio femminile, come nel caso di Polissena strappata alla madre Ecuba e sacrificata dai Greci o Ifigenia sottratta a Clitennestra, e uccisa per permettere alla flotta di lasciare Aulide. Nell'Elettra di Euripide troviamo, forse, l'esempio estremo, perché ad interrompere questa linea matriarcale e a compiere il matricidio che nella tradizione era affidato ad Oreste, è Elettra che, senza scrupolo alcuno, uccide la madre. Significativo è come il matricidio avviene poiché Elettra manda a chiamare la madre facendole credere che ha appena partorito, ed è a quel punto che la uccide. Quando la discendenza femminile sembra continuare, invece, si interrompe.

${ }^{2}$ Un passaggio decisivo nel processo di formazione del patriarcato e di una genealogia maschile, è rappresentato del controllo e dall'appropriazione da parte degli uomini della funzione generativa e procreativa. Tra i miti che vogliono cancellare il ruolo di generatrice della madre troviamo il mito assirobabilonese contenuto nell'Enuma Elish (l'Epopea della Creazione) del 1750 a.C., che racconta la lotta tra Marduk e Tiamat, e i miti legati alla figura di Zeus che da solo crea la vita, generando Dioniso dalla sua coscia e Atena dalla sua testa.

${ }^{3}$ La ricostruzione di una genealogia ed in particolare la riflessione riguardante la relazione madrefiglia rappresenta uno dei temi cardini all'interno del dibattito del femminismo degli anni 70'-80', prima in Francia, ma anche in Italia. Due sono i testi fondamentali: "Il corpo a corpo con la madre" di Luce Irigaray, contenuto in Sessi e genealogie, pubblicato nel 1987, e L'ordine simbolico della madre di Luisa Muraro, pubblicato in Italia nel 1991.
} 
quest'ultima, Zeus. Demetra, disperata, va in cerca della figlia e, non trovandola, in quanto dea della fertilità decide di interrompere la crescita dei raccolti, dei frutti e dei fiori. Alla fine, Ade acconsente che Persefone torni con la madre sei mesi (primavera ed estate) in modo che la natura possa continuare a fare il suo corso.

Irigaray interpreta il mito come la volontà di opporsi e spezzare quello che rappresenta il rapporto più evoluto ed etico. Nella relazione madre-figlio non c'è reciprocità, poiché il figlio non può identificarsi con chi l'ha generato e portato in grembo. Nella relazione madre-figlia questa reciprocità è possibile poiché la figlia a sua volta è potenzialmente madre.

Nelle autrici analizzate, la scrittura si presenta come uno strumento ideale per intrecciare e innestare relazioni matrilineari, per costruire e ricostruire legami perduti o che non si sono mai generati. Scrivere è l'inizio di un viaggio verso l'altra, ma anche verso se stesse, è un camminare a ritroso alla ricerca di tracce del proprio presente.

La riflessione sul rapporto con la madre, ma anche con altre figure femminili della famiglia rappresenta una delle tematiche che trova maggiormente spazio nella letteratura delle scrittrici del Novecento, anche attraverso il genere del romanzo autobiografico. Si pensi ad esempio a Una donna (1906) di Sibilla Aleramo, Menzogna e sortilegio (1948) di Elsa Morante, Lessico famigliare (1963) di Natalia Ginzburg, Madre e figlia (1981) di Francesca Sanvitale, L'amore molesto (1992) e La figlia oscura (2006) di Elena Ferrante fino al recente Tre donne (2017) di Dacia Maraini. ${ }^{4}$.

Va' dove ti porta il cuore (1994) di Susanna Tamaro, Mal di pietre (2006) di Milena Agus e Accabadora (2009) di Michela Murgia, rappresentano non solo tre esempi di costruzione di genealogie femminili atipiche, e nel caso di Murgia non di sangue, ma evidenziano il tentativo e la necessità delle protagoniste di comunicare/comunicarsi. L'avvicinamento tra donne propizia la pratica della scrittura, come osserva Helene Cixous (1997) in un gioco di specchi e rimandi, dove scrivere sulle altre diventa scrivere su se stesse.

Va' dove ti porta il cuore di Susanna Tamaro edito nel 1994 è stato uno dei primi romanzi a dare visibilità letteraria ai rapporti tra donne, riuscendo a richiamare l'attenzione della critica e del pubblico ed offrendo una nuova interpretazione delle relazioni femminili all'interno della famiglia e della società.

Il romanzo si sviluppa attraverso una lunga lettera della protagonista Olga alla nipote con la quale cerca di curare la ferita provocata dalla morte prematura della figlia. In questa lettera la nonna, colpita da un ictus, decide di confidare alla nipote Marta tutti i segreti del suo passato, per provare a ricucire il loro rapporto che con il tempo si è fatto sempre più difficile. Dietro alla decisione di Olga di iniziare

\footnotetext{
${ }^{4}$ Alcuni di questi testi vengono analizzati in S. Chemotti (2009), L'inchiostro bianco. Madri e figlie nella narrativa italiana contemporanea, Padova: Il Poligrafo.
} 
a scrivere a Marta, c'è la volontà di lasciare un segno concreto per costruire una discendenza, creare sulla carta quel legame che non è riuscita ad avere durante la sua vita con sua madre e sua figlia.

I giorni passavano e non prendevo nessun tipo di decisione. Poi questa mattina il suggerimento della rosa. Scrivile una lettera, un piccolo diario dei tuoi giorni che continui a tenerle compagnia. E così eccomi qua, in cucina, con un tuo vecchio quaderno davanti a mordicchiare la penna come un bambino in difficoltà con i compiti. Un testamento? Non proprio, piuttosto qualcosa che ti segua negli anni, qualcosa che potrai leggere ogni volta che sentirai il bisogno di avermi vicina. Non temere, non voglio pontificare, né rattristarti, soltanto chiacchierare un po' con l'intimità che ci legava una volta e che, negli ultimi anni, abbiamo perso. Per avere a lungo vissuto e aver lasciato dietro di me tante persone, so ormai che i morti pesano non tanto per l'assenza, quanto per ciò che tra loro e noi - non è stato detto (Tamaro, 1994, 15).

Attraverso il suo diario-lettera, Olga ripercorre le varie tappe della sua relazione con la nipote, dai momenti di affetto e intimità, fino al graduale distacco ed allontanamento. Scrive a Marta, ma anche a se stessa e, poco a poco, una parola dopo l'altra, la scrittura la aiuta a interpretare la sua relazione con le altre donne della sua famiglia, la figlia Ilaria e sua madre.

Il legame con la madre è rimasto come incompiuto e Olga ricorda con sofferenza l'incapacità di creare un dialogo con lei. Non c'è, però, nessuna condanna nelle sue parole ma piuttosto un tentativo di interpretare il suo comportamento come una conseguenza delle norme e dalle regole imposte dalla società. Ed è proprio ricordando i racconti della madre, ormai anziana, che riesce a perdonarla e in qualche modo a riconciliarsi con lei.

Soltanto da vecchia mia madre ha cominciato a raccontarmi qualcosa della sua infanzia. Sua madre era morta quando lei era ancora bambina, prima di lei aveva avuto un maschio stroncato a tre anni da una polmonite. Lei era stata concepita subito dopo e aveva avuto la sventura di nascere non solo femmina ma anche il giorno stesso in cui suo fratello era morto. Per ricordare questa triste coincidenza, fin da lattante era stata vestita con i colori del lutto. Sulla sua culla troneggiava un grande ritratto ad olio del fratello. Serviva a farle presente, ogni volta che apriva gli occhi, di essere solo un rimpiazzo, una copia sbiadita di qualcuno migliore. Capisci? Come incolparla allora della sua freddezza, delle sue scelte sbagliate, del suo essere lontana da tutto? [...] L'infelicità abitualmente segue la linea femminile. Come certe anomalie genetiche, passa di madre in figlia. Passando, invece di smorzarsi, diviene via via più intensa, più inestirpabile e profonda (Tamaro, 1994, 40).

Olga cerca di percorrere quella strada che non ha avuto il coraggio di intraprendere con la figlia Ilaria e in un certo modo è anche a lei che scrive attraverso l'immagine riflessa di Marta: "Questa lettera avrei dovuto scriverla a tua madre, invece l'ho scritta a te. Se non l'avessi scritta per niente allora sì che la mia esistenza 
sarebbe stata davvero un fallimento. Fare errori è naturale, andarsene senza averli compresi vanifica il senso di una vita" (Tamaro, 1994, 142).

Alla fine, la linea matriarcale che appariva perduta, sembra potersi ricomporre attraverso un nuovo inizio. Olga si reca in soffitta e ritrova uno stampo da torta che rappresenta un lascito delle varie generazioni di donne della sua famiglia:

Questo stampo apparteneva a mia nonna cioè alla tua trisavola ed è l'unico oggetto rimasto in tutta la storia femminile della nostra famiglia. [...] Pensa quante volte nella sua esistenza è entrato e uscito dal forno, quanti forni diversi e sempre più moderni ha visto, quante mani diverse eppure simili hanno riempito l'impasto. L'ho portato giù per farlo vivere ancora, perché tu lo usi e magari, a tua volta, lo lasci in uso alle tue figlie, perché nella sua storia di oggetto umile riassuma e ricordi la storia delle nostre generazioni (Tamaro, 1994, 159-160).

La genealogia tracciata dalla protagonista di $\mathrm{Va}$ ' dove ti porta il cuore, sebbene possa apparire una genealogia in parte rassegnata, scandita dall'autocommiserazione e autoreferenziale, trova un riscatto nella scrittura, nelle parole affidate alla nipote. Olga riflette e dialoga con il femminile della sua famiglia e in questo modo rompe il silenzio e l'incomunicabilità sentimentale in cui le donne sono state confinate e costrette.

Anche Milena Agus presenta una nonna e una nipote che, attraverso il racconto, rielaborano e ricostruiscono il loro vissuto e riescono a ricavarne una grande forza che incide ed influenza il loro presente. In Mal di pietre si ribalta il vincolo nonnanipote presente in $\mathrm{Va}$ ' dove ti porta il cuore. È la nipote, attraverso un quaderno, dove la nonna appuntava le sue vicende e i suoi pensieri, a raccontarne le vicende della gioventù, il matrimonio che sembra sfumare per il carattere ribelle e anticonformista e la ricerca costante dell'amore. Alla fine il matrimonio arriva con un vedovo, che giunge a Cagliari nel '43. È un'unione senza amore, e il vero innamoramento si realizzerà solo grazie al 'mal di pietre', durante un viaggio in Continente, per una cura termale.

Il titolo allude infatti ai calcoli renali di cui soffre la nonna e contemporaneamente agli ostacoli che durante la vita deve affrontare. Presso le terme conoscerà il Reduce, sopravvissuto alla guerra e mutilato, uomo colto e gentile, con cui condividerà poesie e musica. Dopo questo viaggio non si incontreranno più, ma dalla loro relazione nascerà il padre della narratrice, destinato a diventare un grande concertista.

Come in $\mathrm{Va}$ ' dove ti porta il cuore, anche in Mal di pietre la parola scritta svolge un ruolo fondamentale. Tutto il romanzo è scandito dall'amore della nonna per la scrittura, prima negata e proibita ma che poi le permette di spezzare le regole e superare il suo stato di isolamento. Attraverso un'efficace struttura a cornice, le parole della nonna si intrecciano con quelle della nipote in un racconto che unisce le loro vite attraverso continui riferimenti. La genealogia femminile familiare 
diventa anche una genealogia letteraria dove la scrittura funziona come eredità e discendenza, non solo di sangue ma anche di parole. L'incontro con il Reduce significa anche riconciliarsi con quella scrittura che aveva causato il ritardo nel suo matrimonio e che la famiglia voleva sopprimere e che ora, invece, può ritrovare.

Lei non aveva mai avuto uno scrittoio, né aveva mai potuto sedersi a un tavolo, perché scriveva sempre di nascosto, con il quaderno in grembo che nascondeva appena sentiva arrivare qualcuno.

Sullo scrittoio c'era una cartella di pelle con dentro tanti fogli di carta intestata, una boccetta di inchiostro, una penna con il pennino e la carta assorbente. Allora nonna la prima cosa che fece, prima ancora di togliersi il cappotto, fu di tirare fuori dalla valigia il suo quaderno e di metterlo in pompa magna sullo scrittoio, dentro la cartella di pelle, poi chiuse la porta bene a chiave per la paura che qualcuno entrasse all'improvviso e vedesse cosa c'era scritto nel quaderno (Agus, 2006, 28).

Il rapporto con la nonna è rivendicato con orgoglio dall'autrice che vuole raccoglierne il testimone, ed è proprio la sua storia a guidarla e darle la forza di scrivere.

Io sono nata che mia nonna aveva più di settant'anni. Mi ricordo che da piccola la trovavo bellissima e stavo sempre incantata a vedere quando si pettinava e si faceva sa crocchia all'antica, con le trecce di capelli che non sono mai diventati bianchi, né radi, e che partivano dalla discriminatura in mezzo per poi essere raccolti in due chignon. Ero orgogliosa quando veniva a prendermi a scuola con quel sorriso giovane tra le mamme e i padri degli altri, perché i miei essendo musicisti, erano sempre in giro per il mondo. Mia nonna è stata tutta per me, almeno quanto mio padre tutto per la musica e mia madre tutta per mio padre (Agus, 2006, 23).

Accabadora (2009) di Michela Murgia, racconta la storia di Maria Listru, una "fillus de anima", che è come vengono chiamati i bambini generati due volte, dalla povertà di una donna e dalla sterilità di un'altra, come chiarisce la stessa autrice:

[...] sono sempre stata affascinata dalle relazioni famigliari che non hanno il sangue come discriminante. Il tema della maternità elettiva mi appartiene profondamente, perché a mia volta sono fill'e anima; ma per non cadere nella trappola del parallelismo autobiografico, ho scelto volutamente di narrare il rapporto dal punto di vista della madre adottiva, una figura per la quale accompagnare i destini a compimento è solo una delle possibili sfumature della sua maternità, non necessariamente la più oscura (Murgia, 2009).

La protagonista viene presa con sé dall'accabadora Bonaria Urrai. Un'eutanasista ante litteram, che nella cultura sarda è 'l'ultima madre' che aiuta il destino a compiersi. Il legame tra Tzia Bonaria e Maria ha inizio quando l'accabadora vede la piccola rubare delle ciliegie in un negozio e, rendendosi conto che nessuno si occupa di lei, decide di adottarla. Maria, grazie a questo incontro capirà come nella vita, oltre alla madre naturale, se ne possano incontrare altre lungo il proprio cammino. 
Maria, tu di chi sei figlia? [...].

- Di Anna Teresa e Sisinnio Listru [...].

- Giusto. E però dove vivi? [...].

- Vivo qui con voi, Tzia.

- Quindi vivi staccata da tua madre, ma sei sempre sua figlia. È così? Non vivete insieme, ma siete madre e figlia [...].

- Siamo mamma e figlia, sì... ma non proprio una famiglia. Se eravamo una famiglia, non si metteva d'accordo con voi... cioè, io credo che voi siete la mia famiglia. Perché noi siamo più vicine (Murgia, 2009, 24-25).

A fianco della piccola Maria, Bonaria mostra nuovi sentimenti e sensazioni, riuscendo a recuperare una dimensione della propria intimità-affettività, che in seguito alla scomparsa del marito aveva totalmente cancellato, nascondendosi e rifugiandosi in un lutto perenne.

Con un gesto istintivo prese la testa della ragazzina e se la strinse al petto come per scaldarla. Sciocca che sei, Mariedda Listru! Tu sei diventata mia figlia nel momento stesso in cui ti ho visto, e non sapevi nemmeno chi ero [...] Non l'aveva mai chiamata così, e non lo fece mai più in quel modo. Ma a Maria quel piacere denso, così simile ad un dolore in bocca, rimase impresso per molto tempo (Murgia, 2009, 26).

Quando Maria si rende conto di cosa fa in realtà la madre adottiva mentre si assenta di notte, non riesce ad accettarlo, nonostante Tzia Bonaria cerchi di farle capire come 'nessuno si fa da solo e nessuno da solo si disfa', e di come vita e morte si rincorrano avvolte nell'ineluttabilità del destino.

- Se le cose devono accadere, al momento giusto accadono da sole.

- Sei nata tu forse da sola, Maria? Sei uscita con le tue forze dal ventre di tua madre? [...] Ti sei tagliata da sola il cordone? Non ti hanno forse lavata e allattata? Non sei nata e cresciuta due volte per grazia di altri, o sei così brava che hai fatto tutto da sola? [...] Altri hanno deciso per te, e altri decideranno quando servirà di farlo. Non c'è nessun vivo che arrivi al suo giorno senza aver avuto padri e madri ad ogni angolo di strada, Maria, e tu dovresti saperlo più di tutti (Murgia, 2009, 117).

Le parole di Tzia Bonaria sembrano richiamarsi ed evocare il simbolo dell'Uroboro, rappresentato come un serpente che si morde la coda creando un cerchio senza fine. Strettamente collegato alla Grande Madre, ne evidenzia la continuità ma anche la dualità. D'altronde la Grande Madre è costituita da elementi opposti: la madre buona che offre protezione, nutrimento e calore e la madre terribile che si mostra distruttrice. Le parole di Bonaria diventano una confessione e al tempo stesso una profezia che accompagnerà Maria segnandone il destino.

- Non mi si è aperto il ventre, proseguì, e Dio sa se l'avrei voluto, ma ho imparato da sola che ai figli bisogna dare lo schiaffo e la carezza, e il seno, e il vino della festa. Anche io avevo la mia parte da fare, e l'ho fatta. 
- E quale parte era?

- L'ultima. Io sono stata l'ultima madre che alcuni hanno visto [...].

- Per me siete stata la prima, e se mi chiedeste di morire, io non sarei capace di uccidervi solo perché e quello che volete [...].

- Non dire mai: di quest'acqua no ne bevo. Potresti trovarti nella tinozza senza sapere come ci sei entrata $[\ldots]$.

- Quando verrà il momento, Maria, scoprirai cose di te che non conosci ancora (Murgia, 2009, 117-118).

La protagonista lascerà la Sardegna, per rifugiarsi a Torino, dove lavorando come baby sitter, cercherà di dimenticare Bonaria e il mondo che rappresenta, interrompendo il vincolo matriarcale. Tornata a casa per prendersi cura della mamma adottiva malata, capirà il significato delle sue parole e rifletterà sulla necessità di accogliere la vita ma anche la morte. Accompagnerà Tzia Bonaria nel suo ultimo viaggio comprendendone la missione e raccogliendone, forse, il testimone.

Entrando in camera trovò il cuscino in attesa sulla poltrona accanto al letto e lo prese, poi si avvicinò con la certezza che stavolta nessun senso di colpa l'avrebbe fermata. Forse fu il gesto di tenerezza che aveva visto compiere ad Andría a spingerla a chinare il capo verso il volto di Bonaria prima di agire, sfiorandole la guancia con le labbra con una levità che non sentiva di aver mai avuto da quando era tornata a casa. Ci sono cose che si sanno e basta e le prove sono solo conferma, fu con l'ombra netta di una intuizione che Maria Listru seppe con certezza che sua madre Bonaria Urrai era morta (Murgia, $2009,162)$.

In tutti e tre i romanzi si assiste ad un intreccio di vite e parole, nel quale si disordina la linea temporale passato/presente, ma anche la relazione di ascendenza/discendenza, per creare una sororità che fonde tempo, spazio e narrazioni diverse. Nell'ampiezza della scrittura autobiografica, le autrici e/o protagoniste restituiscono al femminile la funzione simbolica di generare non solo la vita ma anche la creatività attraverso la scrittura. In questo senso, il recupero della lingua della madre, del suo racconto e della sua dimensione simbolica permette di abbattere le barriere della solitudine patriarcale e di rigenerare una genealogia matrilineare. 


\section{BIBLIOGRAFIA}

Agus, M. (2006). Mal di pietre. Milano: Edizioni Nottetempo.

Chemotti, S. (2009). L'inchiostro bianco. Madri e figlie nella narrativa italiana contemporanea. Padova: Il Poligrafo.

Cixous, H. (1997). Il riso della medusa. In AA.VV., Critiche femministe e teorie letterarie (pp. 221-246). Bologna: Clueb.

Diotima. (2007). L'ombra della madre. Napoli: Liguori.

Fanning, U. (2013). Maternal Prescriptions and Descriptions in Post-Unification Italy. In K. Mitchell, H. Sanson (Eds.), Women and Gender in Post-Unification Italy: Between Private and Public Spheres (pp. 13-37). Oxford: Peter Lang.

Freixas, A. (Ed.). (1996). Madres y hijas. Barcelona: Anagrama.

Giorgio, A. (Ed.). (2002). Writing mothers and Daughters. Renegotiating the Mother in Western European Narratives by Women. New York: Berghahn Books.

Irigaray, L. (1989). Sessi e genealogie, trad. it di L. Muraro. Milano: La Tartaruga.

Muraro, L. (1991). L'ordine simbolico della madre. Roma: Editori Riuniti.

Murgia, M. (2009). Accabadora. Torino: Einaudi.

Rodriguez Magda, R. (1999). Foucault y la genealogía de los sexos. Barcelona: Anthropos.

Scacchi, A. (Ed.). (2005). Lo specchio materno. Madri e figlie tra biografia e letteratura. Roma: Sossella.

Tamaro, S. (1994). Va' dove ti porta il cuore. Milano: Baldini e Castoldi. 